\title{
2-D NUMERICAL WAVE FLUME WITH SOLID-GAS-LIQUID INTERACTION AND ITS APPLICATION
}

\author{
Koji Kawasaki ${ }^{1}$, Yoshitaka Takasu² and Han Dinh Ut ${ }^{3}$
}

\begin{abstract}
A 2-D numerical wave flume based on a multiphase flow model with solid-gas-liquid interaction is presented in this paper. The governing equations are divided into an advection step and a non-advection step by making use of a time splitting method. A CIP method is used to calculate the hyperbolic equations for velocity and pressure at the advection step, while equations at the non-advection step are solved with an extended SMAC method. Conservation equation of mass is directly solved by using a CIP-CSL2 method. A non-reflective wave generator employing a source/sink method for wave generation and an energy dissipation zone are utilized to realize the numerical wave flume. Besides, the constitutive laws of the non-Newtonian fluid are taken into account to make the model capable of simulating the behavior of Bingham fluid. The validity and utility of the numerical wave flume are demonstrated by applying it to wave breaking and post-breaking wave deformation on the slope, the dynamic motion of a floating body under wave action and the collapse of the Bingham fluid column with multiple rigid bodies.
\end{abstract}

Keywords: 2-D numerical wave flume; CIP method; CIP-CSL2 method; extended SMAC method; non-reflective wave generator; Bingham fluid.

\section{INTRODUCTION}

Coastal zones are at risk of huge coastal disasters caused by tsunami, storm surge, extreme wave, wave overtopping and so on. A better understanding of these complicated mechanism processes with air-water-structure interaction is of extremely importance from the viewpoint of disaster prevention. Various theoretical, experimental and numerical studies have been conducted for investigating and clarifying physical phenomena in coastal areas. However, these physical phenomena are generally constituted by solid, gas and liquid phase fields, such as structures, sediments, winds, waves and currents. Therefore, it would be generally difficult to predict multiphase fields with high accuracy. For example, tsunami disasters cause not only direct damages due to tsunami itself but also indirect damages resulting from collisions between coastal defense facilities and drifting bodies including timbers, containers and cars.

Recently, numerical simulation approaches have been widely used as one of the tools to resolve complicated coastal hydraulic phenomena with nonlinear interactions among solid, gas and liquid phases and prevent coastal disasters, with the advance of computer performance and the development of higher-order numerical schemes. For instance, Kawasaki (2005a) and Kawasaki (2005b) have developed two- and three-dimensional numerical models of multiphase flow by employing a CIP (Constrained Interpolation Profile) method and an extended SMAC (Simplified Marker And Cell) method in order to precisely analyze complex physical phenomena with solid-gas-liquid interaction and provide useful information in designing coastal structures. Kawasaki and Hakamata (2006) proposed a new two-dimensional numerical model of solid-gas-liquid phase flows by improving the computational algorithm to resolve mass conservation problem and introducing a LES (Large Eddy Simulation) based on the Smagorinsky model. Furthermore, Kawasaki and Mizutani (2007) confirmed the utility and validity of the model by comparing the numerical results with the experimental ones regarding wave pressures acting on rigid bodies under bore action, which was induced by the collapse of a water column. However, the model has been limited to the numerical analysis of dynamic behavior of only one rigid body. Kawasaki and Ogiso (2009) developed a three-dimensional solid-gas-liquid phase flow model so as to analyze the dynamic behavior of multiple rigid bodies. The previous models have achieved in some applications, but still have some problems relating to the conservation of mass. These models also have not had the functions of a numerical wave flume including wave generator and non-reflective boundary treatment.

Reclamation lands in coastal zones under liquefaction conditions due to strong earthquake would be deformed in a similar behavior to a Bingham fluid, which is one of the non-Newtonian fluids.

\footnotetext{
${ }^{1}$ Associate professor, Department of Civil Engineering, Nagoya University, C1-3(651), Furo-cho, Chikusa-ku, Nagoya Aichi 464-8603, Japan

${ }^{2}$ Master student, Department of Civil Engineering, Nagoya University, C1-3(651), Furo-cho, Chikusa-ku, Nagoya, Aichi 464-8603, Japan

${ }^{3}$ Doctoral student, Department of Civil Engineering, Nagoya University, C1-3(651), Furo-cho, Chikusa-ku, Nagoya, Aichi 464-8603, Japan
} 
Consequently, the constitutive laws of the non-Newtonian fluids should also be considered in the numerical model.

Based on the above-mentioned, the purpose of this study is to develop a two-dimensional (2-D) numerical wave flume with solid-gas-liquid interaction. A constitutive law for Bingham fluid is also introduced into the model so as to be able to analyze non-Newtonian fluid dynamics. A LES-based DTM (Dynamic Two-parameter Mixed) turbulence model, the third-order Adams-Bashforth scheme and a CIP-CSL2 (Constrained Interpolation Profile - Conservative Semi-Lagrangian 2) method for the conservation equation of mass are incorporated in order to enhance the computation accuracy. Furthermore, a non-reflective wave generator employing a source/sink method for wave generation (Brorsen and Larsen, 1987) and an energy dissipation zone (Cruz et al., 1993) are employed to realize a numerical wave flume.

\section{2-D NUMERICAL WAVE FLUME WITH SOLID-GAS-LIQUID INTERACTION}

\section{Governing Equations}

The governing equations consist of the conservation equation of mass (Eq. (1)), the Navier-Stokes equations (Eq. (2)), the pressure equation for compressible fluid (Eq. (3)), the advection equation of density functions (Eq. (4)) and the equation of state for barotropic fluid (Eq. (5)). The equations allow us to precisely compute not only incompressible but also compressible multiphase flows.

$$
\begin{aligned}
& \frac{\partial \rho}{\partial t}+\frac{\partial\left(u_{j} \rho\right)}{\partial x_{j}}=\rho q \\
& \frac{\partial u_{i}}{\partial t}+u_{j} \frac{\partial u_{i}}{\partial x_{j}}=-\frac{1}{\rho} \frac{\partial p}{\partial x_{i}}-g_{i}+\frac{f_{s i}}{\rho}+\frac{\partial}{\partial x_{j}}\left(-\tau_{i j}+2 \frac{\mu}{\rho} S_{i j}\right)-D_{i} u_{i} \\
& \frac{\partial p}{\partial t}+u_{j} \frac{\partial p}{\partial x_{j}}=-\rho C_{l s}^{2}\left(\frac{\partial u_{j}}{\partial x_{j}}-q\right) \\
& \frac{\partial \phi_{I}}{\partial t}+u_{j} \frac{\partial \phi_{I}}{\partial x_{j}}=0 \\
& \rho=f(p) \\
& q=\left\{\begin{array}{l}
0 \quad\left(x \neq x_{s}\right) \\
q^{*}\left(x=x_{s}\right)
\end{array}\right.
\end{aligned}
$$

where, $x_{i}$ is position vector $(x, z), u_{i}$ is velocity component in the direction of $i, \rho$ is fluid density, $p$ is pressure, $g_{i}$ is gravitational acceleration vector $(0,-g), f_{s i}$ is surface tension term, $\tau_{i j}$ is turbulence term, $\mu$ is the coefficient of viscosity, $S_{i j}$ is strain rate tensor $\left(\partial u_{i} / \partial x_{j}+\partial u_{j} / \partial x_{i}\right), D_{i}$ is dissipation coefficient used in energy dissipation zones, $C_{l s}$ is local sound speed, $t$ is time and $\phi_{I}(I=1 \sim 3)$ are density functions for respective phases ( $\phi_{1}$ : solid phase; $\phi_{2}$ : liquid phase; $\phi_{3}$ : gas phase) that represent the rate of fractional volume for each phase in a cell and these functions need to satisfy the relationship: $\phi_{1}+\phi_{2}+\phi_{3}=1\left(0 \leq \phi_{I} \leq 1\right)$ in a cell. $q=q(z, t)$ is wave generation source with its strength $q^{*}$ assigned only at source line $\left(x=x_{s}\right)$.

\section{Computational Algorithm}

Fig. 1 indicates the computational flow chart of the 2-D numerical wave flume. Eqs. (2) and (3) are divided into an advection step and a non-advection step by making use of a time splitting method as shown in Eqs. (7) (10). The resultant equations are discretized by employing irregular staggered mesh grids. A CIP method developed by Yabe and Aoki (1991) is used to calculate the hyperbolic equations for all variables at the advection step, while equations at the non-advection step are solved with an extended SMAC method, which can simulate both compressible and incompressible fluid. Eq. (1) is solved by a CIP-CSL2 method proposed by Nakamura et al. (2001), which is one of the conservative methods extended from a CIP method. The effect of surface tension on the gas-liquid interface is evaluated by using a CSF (Continuum Surface Force) model proposed by Brackbill et al. (1992), which interprets surface tension as a continuous mass force across the interface. A LES-based on a DTM model developed by Salvetti et al. (1995) is applied for estimating turbulence quantities. 


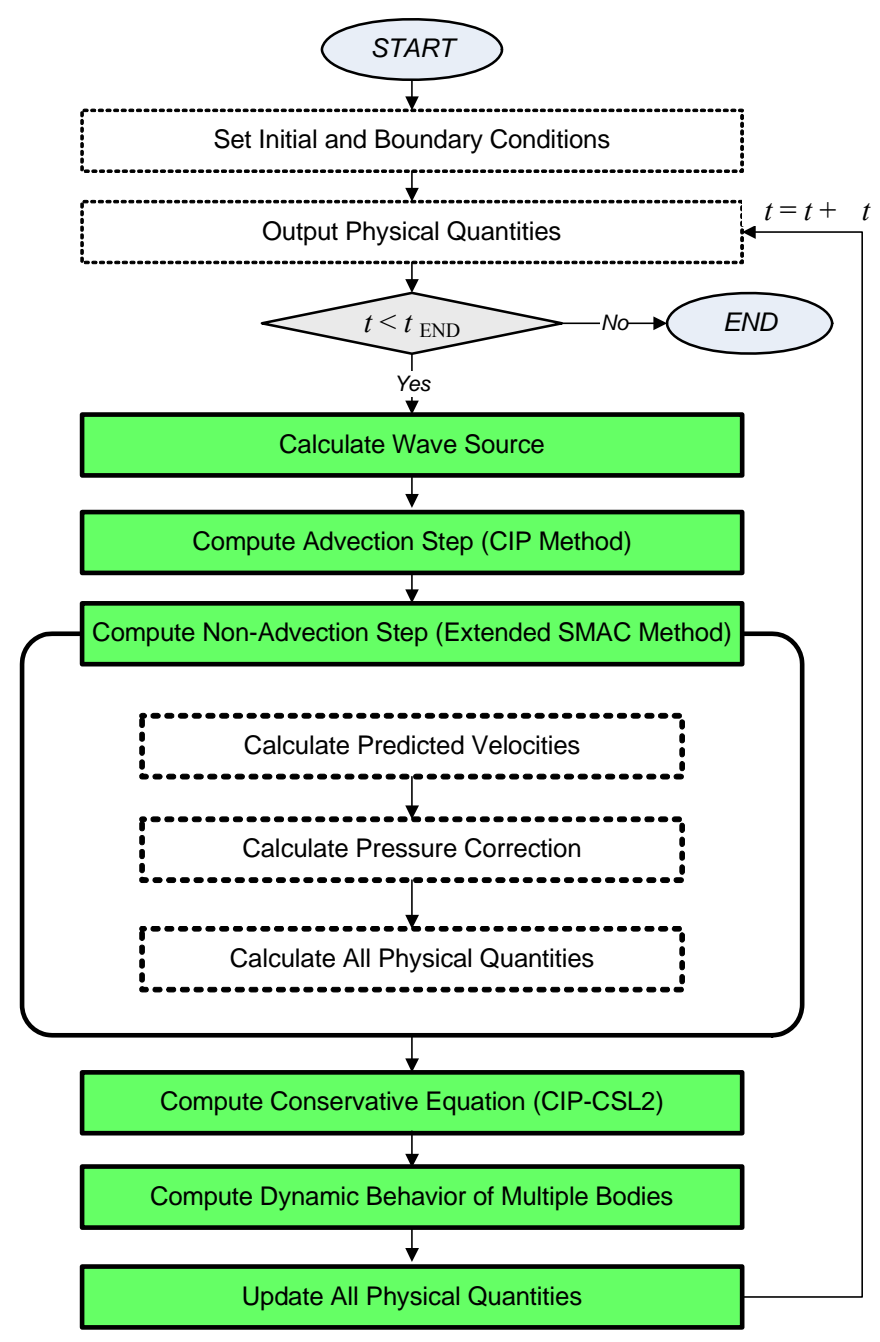

Figure 1. Flow chart of multiphase flow model

[Advection step]

$$
\begin{aligned}
& \frac{\partial u_{i}}{\partial t}+u_{j} \frac{\partial u_{i}}{\partial x_{j}}=0 \\
& \frac{\partial p}{\partial t}+u_{j} \frac{\partial p}{\partial x_{j}}=0
\end{aligned}
$$

[Non-advection step]

$$
\begin{aligned}
& \frac{\partial u_{i}^{n+1}}{\partial t}=-\frac{1}{\rho^{n}} \frac{\partial p^{n+1}}{\partial x_{i}}+F_{i} \\
& \frac{\partial p^{n+1}}{\partial t}=-\rho C_{l s}{ }^{2}\left(\frac{\partial u_{j}^{n+1}}{\partial x_{j}}-q\right)
\end{aligned}
$$

where, $F_{i}$ represents external force term such as gravity, viscous, surface tension and dissipation zone terms. 


\section{Numerical procedure at advection step}

The equations at the advection step are calculated by using the CIP method with 3rd-order accuracy, which can solve the advection equation precisely, taking advantage of the hyperbolic equation $f$ as represented in Eq. (11).

$$
\frac{\partial f}{\partial t}+u_{j} \frac{\partial f}{\partial x_{j}}=0
$$

Fig. 2 shows the concept of one-dimensional CIP method, in which the spatial distribution of value is interpolated in the range of the interval $\left[x_{i-1}, x_{i}\right]$ with a cubic polynomial function formed as Eq. (12).

$$
F_{i}^{n}(x)=a_{1} X^{3}+a_{2} X^{2}+a_{3} X+a_{4}
$$

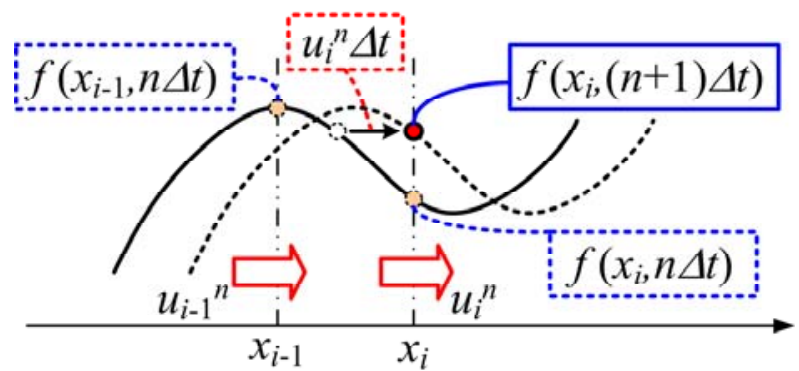

Figure 2. Concept of one-dimensional CIP method

The unknown coefficient $a_{1} \sim a_{4}$ in the interpolation function of $F_{i}^{n}(x)$ are determined from the continuities of $f$ and its spatial derivatives $f_{x}$ at the grid points $x_{i-1}$ and $x_{i}$. Then, value $f_{i}^{*}$ at the next time step is computed by Eq. (13).

$$
f_{i}^{*}=F_{i}^{n}\left(x_{i}-u_{i} \Delta t\right)
$$

\section{Numerical procedure at non-advection step}

Eqs. (9) and (10) cannot be solved explicitly since the unknown variables at the next time step are included in both the left and the right sides of the equations. In the model, the extended SMAC method is used to compute both compressible and incompressible fluids.

The predicted velocity $\tilde{u}_{i}$ is computed explicitly by Eq. (14) with the help of variables after the advection step.

$$
\frac{\tilde{u}_{i}-u_{i}^{*}}{\Delta t}=-\frac{1}{\rho} \nabla p^{*}+F_{i}^{*}
$$

where, superscript $*$ represents the time step after the advection step.

The simultaneous equation for the pressure correction $\delta p=p^{n+1}-p^{*}$ in Eq. (15) is derived by eliminating $\nabla \cdot u_{i}{ }^{n+1}$ from Eq. (10) using Eqs. (9) and (14).

$$
\nabla\left(\frac{1}{\rho^{*}} \nabla \delta p\right)=\left(\frac{\delta p}{\rho^{*} C_{l s}^{2} \Delta t^{2}}-q\right)+\frac{1}{\Delta t} \frac{\partial \tilde{u}_{i}}{\partial x_{i}}
$$

The pressure correction $\delta p$ in Eq. (15) is solved by an ILUCGS (Incomplete LU decomposition Conjugate Gradient Squared) method.

Finally, all the variables at the next time step $t=(n+1) \Delta t$ are updated by Eqs. $(16) \sim(18)$.

$$
u_{i}^{n+1}=\tilde{u}_{i}-\frac{\Delta t}{\rho^{*}} \nabla \delta p
$$




$$
\begin{aligned}
& p_{i}^{n+1}=p_{i}^{*}+\delta p \\
& \rho^{n+1}=\rho^{*}-\rho^{*} \nabla \cdot u_{i}^{n+1} \Delta t
\end{aligned}
$$

The local sound speed $C_{l s}$ and the viscous coefficient $\mu$ for each cell are evaluated from Eqs. (19) and (20).

$$
\begin{aligned}
& C_{l s}=\phi_{1} C_{l s 1}+\phi_{2} C_{l s 2}+\phi_{3} C_{l s 3} \\
& \mu=\phi_{1} \mu_{1}+\phi_{2} \mu_{2}+\phi_{3} \mu_{3}
\end{aligned}
$$

where, the subscripts 1, 2 and 3 represent solid, liquid and gas phases, respectively.

\section{Surface tension}

A CSF model developed by Brackbill et al. (1992) is introduced into the Navier-Stokes equations to evaluate the effects of surface tension between gas and liquid phases. The CSF model assumes that the interface between gas and liquid phases, the thickness of which is in fact zero, has some transition ranges, as shown in Fig.3. The surface tension assumed as the body force is denoted as Eq. (21).

$$
f_{s i}=\sigma k \frac{\nabla \phi_{2}}{\left[\phi_{2}\right]} \frac{\phi_{2}}{\left\langle\phi_{2}\right\rangle}
$$

where, $\sigma$ is the coefficient of the surface tension, $k$ is the curvature, $\left[\phi_{2}\right]=1$ and $<\phi_{2}>=1 / 2$.

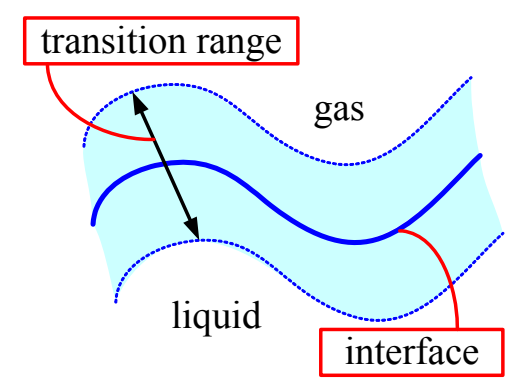

Figure 3. Concept of CSF model

\section{Dynamic motion analysis of multiple rigid bodies}

The density function of solid phase for each rigid body $\phi_{1 l}$ is introduced into the model in order to compute the motions of multiple rigid bodies, in which $l$ represents the number of the bodies. It should be noted that the relationship between $\phi_{1}$ which represents a density function of solid phase in a computational cell and $\phi_{1 l}$ is required to satisfy Eq. (22). First, the motions of rigid bodies are simulated similarly to the numerical procedure proposed by Xiao et al. (1997). Assuming that rigid bodies are a high-viscous fluid, the entire computational domain including the region of rigid bodies is computed in the above-mentioned computational algorithm. However, the distortion of the rigid bodies occurs because they are treated as a fluid. To overcome this problem, the translational velocity $V_{l}$ and angular velocity $\Omega_{l}$ at the mass center of the rigid bodies are computed by Eqs. (23) and (24). Then, the position of each body at the next time step is calculated by applying the computed velocities $U_{l}$, which is the sum of the translational and angular velocities, only to cells in the solid phase. This also indicates that the motions of rigid bodies are simulated without setting any boundary conditions between solid and other phases.

$$
\begin{aligned}
& \phi_{1}=\sum_{l=1}^{L} \phi_{1 l} \leq 1 \\
& \frac{d \boldsymbol{V}_{l}}{d t}=\frac{1}{M_{l}} \int_{V} \frac{d \boldsymbol{u}}{d t} \phi_{1 l} \rho_{s l} d V
\end{aligned}
$$




$$
\begin{aligned}
& \frac{d \Omega_{l}}{d t}=\frac{1}{I_{l}} \int_{V} \boldsymbol{R}_{l} \times \frac{d \boldsymbol{u}}{d t} \phi_{1 l} \rho_{s l} d V \\
& \boldsymbol{R}_{l}=x-x_{0 l} \\
& \boldsymbol{U}_{l}=\boldsymbol{V}_{l}+\Omega_{l} \boldsymbol{R}_{l}
\end{aligned}
$$

where, $L$ is the total number of rigid bodies, $M_{l}$ is total mass, $\rho_{s l}$ is density, $I_{l}$ is the moment of inertia, $R_{l}$ is a position vector from the center point of the body $x_{0 l}$ to an arbitrary location $x$ and $d u / d t$ is calculated using the pressure values within the bodies based on the Newton's second law of motion.

\section{Modeling of Constitutive Law of Bingham Fluid}

As shown in Fig.4, Bingham fluid treated in the present study has similar characteristics to the Newtonian fluid when the shear stress $\tau$ exceeds the yield shear stress $\tau_{y}$. However, it is said to be difficult from the viewpoint of numerical suitability to reproduce well the sudden change of fluid property. Therefore, a bi-viscosity model, which can compute stably by dividing the Bingham fluid into two properties in terms of shear strain rate $S$, was adopted in this study referring Moriguchi et al. (2005). Finally, the influence of the constitutive law of Bingham fluid was considered by substituting the artificial viscosity $\mu$ ' expressed by Eq. (28) into viscosity coefficient $\mu$ in the right hand side of the momentum equation (Eq. (2)).

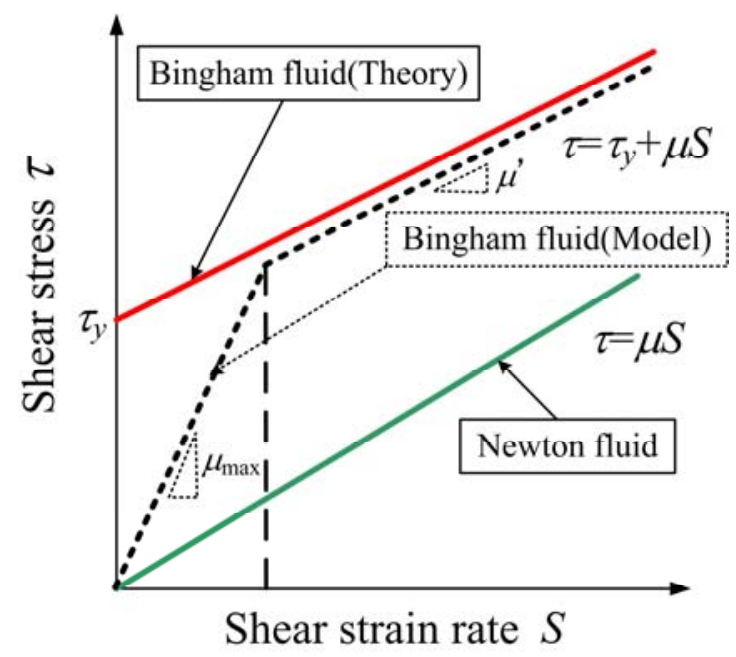

Figure 4. Constitutive low of Bingham fluid

$$
\begin{aligned}
& \tau_{y}=c+p \tan \phi \\
& \mu^{\prime}= \begin{cases}\mu+\frac{\tau_{y}}{|S|} & |S| \geq S_{c} \\
\mu+\frac{\tau_{y}}{\left|S_{c}\right|} & |S|<S_{c}\end{cases}
\end{aligned}
$$

where, $\phi$ is the internal friction angle and $c$ is the cohesion. The critical shear strain rate $S_{c}$ is expressed by Eq. (29) in a similar way to Yamada et al. (1998). In Eq. (29), $\beta$ is an optional constant number and was set to $\beta=0.02$ in this study. The shear strain rate $|S|$ is calculated by Eq. (30).

$$
\begin{aligned}
& S_{c}=\frac{\beta \tau_{y}}{\mu} \\
& |S|=\sqrt{2\left\{\left(\frac{\partial u}{\partial x}\right)^{2}+\left(\frac{\partial w}{\partial z}\right)^{2}\right\}+4\left\{\frac{1}{2}\left(\frac{\partial w}{\partial x}+\frac{\partial u}{\partial z}\right)\right\}^{2}}
\end{aligned}
$$




\section{RESULTS AND DISCUSSION}

\section{Verification of Numerical Wave Flume}

Fig. 5 shows a definition sketch of a 2-D numerical wave flume. The computational domain includes two energy dissipation zones on the left and the right sides of the domain. The origin of $x$ coincides with the wave generation source. In the present simulation, the location of the wave generation source was set at the center of the computational domain. The wave period and wave height are $1.5 \mathrm{~s}$ and $0.1 \mathrm{~m}$ respectively. The time interval $\Delta t$ was set equal to $0.001 \mathrm{~s}$ so that the Courant condition is always satisfied. The detail of numerical conditions was summarized in Table 1.

Fig. 6 shows a comparison between the calculated water surface elevation and the theoretical one with the third-order Stokes theory at some given positions. The numerical results are in good agreement with the theoretical ones. It is also found from Fig. 7 that the waves made by the wave generation source propagate rightward and leftward in the computational domain and are dissipated effectively in the energy dissipation zones. The validity and utility of the numerical wave flume with the non-reflecting wave generator were, therefore, confirmed qualitatively and quantitatively from Figs. 6 and 7.

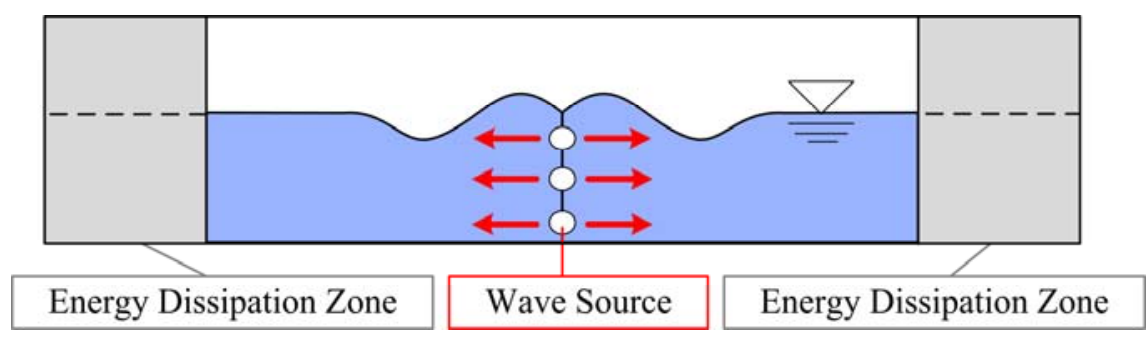

Figure 5. Definition sketch of numerical wave flume

\begin{tabular}{|l|l|l|l|}
\hline Table 1. Numerical conditions (Test 1) \\
\hline Computational domain & $798 \times 60 \mathrm{mesh}$ & Water viscosity coefficient & $1.0 \times 10^{-3}[\mathrm{~Pa} \cdot \mathrm{s}]$ \\
\hline Mesh size & $\Delta x=0.02[\mathrm{~m}] \sim 0.15[\mathrm{~m}]$ & Air viscosity coefficient & $1.8 \times 10^{-5}[\mathrm{~Pa} \cdot \mathrm{s}]$ \\
\cline { 2 - 4 } & $\Delta z=0.02[\mathrm{~m}] \sim 0.08[\mathrm{~m}]$ & Surface tension coefficient & $7.2 \times 10^{-2}[\mathrm{~N} / \mathrm{m}]$ \\
\hline Time step interval & $0.001 \mathrm{~s}$ & Gravitational acceleration & $9.80665\left[\mathrm{~m} / \mathrm{s}^{2}\right]$ \\
\hline Water density & $1000.0\left[\mathrm{~kg} / \mathrm{m}^{3}\right]$ & Atmospheric pressure & $1013[\mathrm{hPa}]$ \\
\hline Air density & $1.20\left[\mathrm{~kg} / \mathrm{m}^{3}\right]$ & & \\
\hline
\end{tabular}



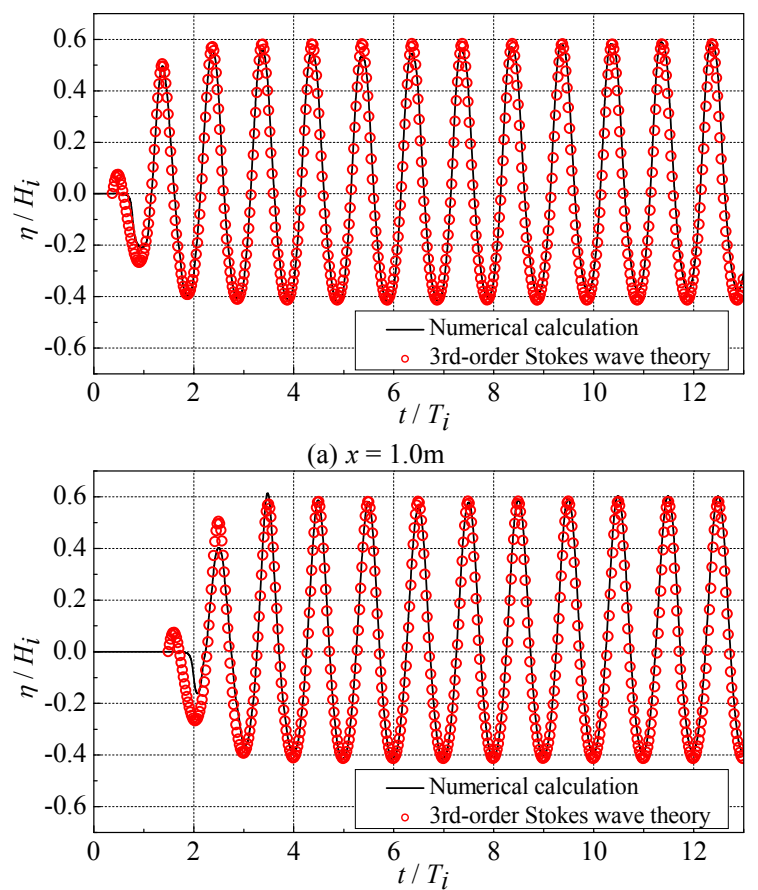

(b) $x=4.0 \mathrm{~m}$

Figure 6. Time variation of water surface elevation 

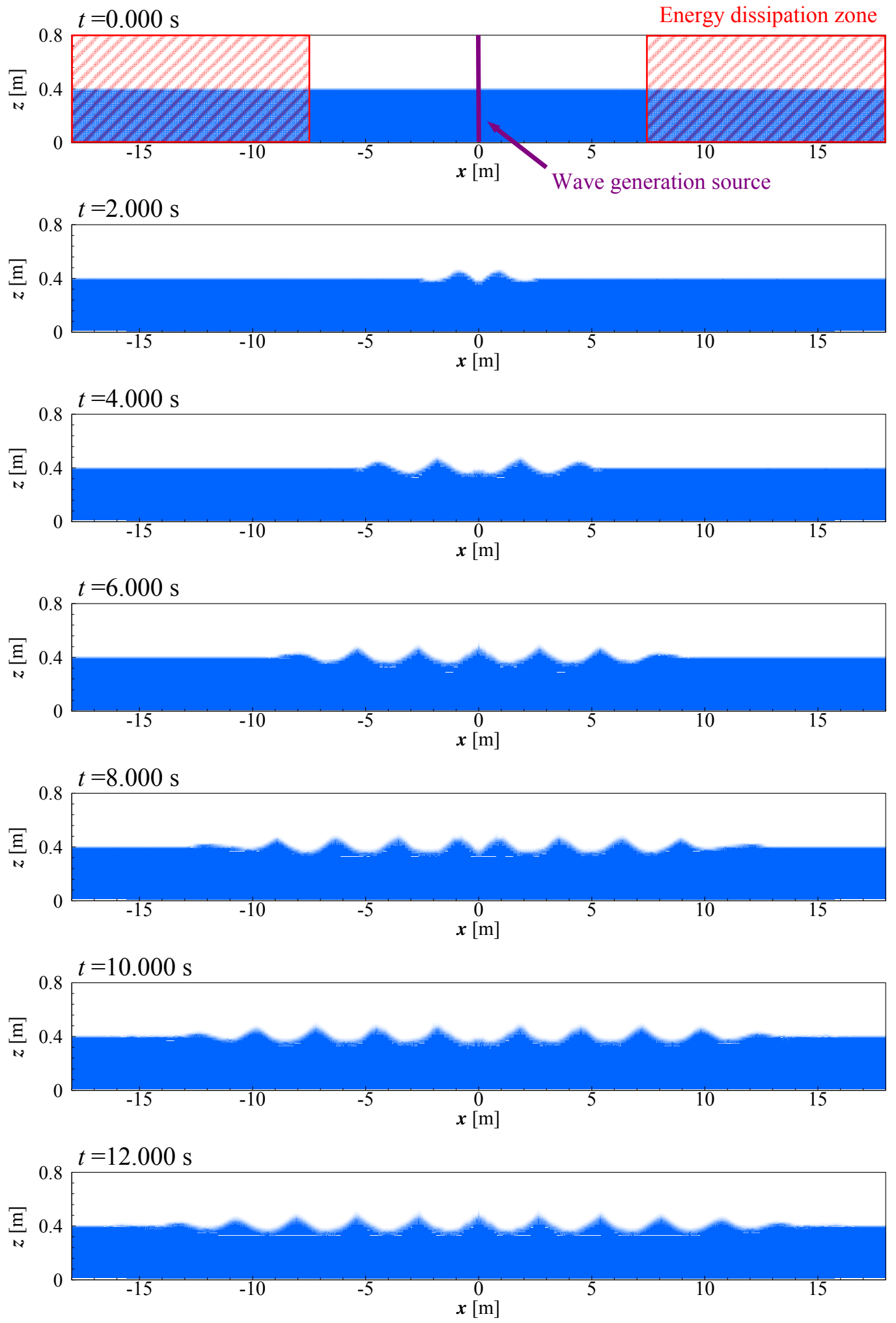

Figure 7. Temporal and spatial variations of water surface 


\section{Wave Breaking Process on a Constant Slope}

In order to verify the validity of the proposed model in treating the interaction of gas and liquid phases, the model is applied to wave breaking phenomenon on a slope, which has been known as a challenging task for numerical simulations because the process includes nonlinear effects and complex interaction between air and water. In the computational domain, the wave generation source was located at $x=0.0 \mathrm{~m}$, and a slope with a constant gradient of $1 / 15$ was set up from $x=10.0 \mathrm{~m}$ to $x=20.0 \mathrm{~m}$. An energy dissipation zone was set from $x=-10.0 \mathrm{~m}$ to $x=0.0 \mathrm{~m}$ to prevent the reflection from the left side of the numerical wave flume. The wave period and wave height are $2.0 \mathrm{~s}$ and $0.15 \mathrm{~m}$, respectively. Detailed numerical conditions are shown in Table 2.

Fig. 8 shows the numerical results of wave breaking process on the slope. The wave generated by the wave source is found to propagate onshore. Then, the wave starts to run up the slope and its amplitude increases significantly due to the effect of wave shoaling. The wave crest subsequently continues to steepen and eventually leads to wave breaking. In Fig. 9, the wave breaking process with overturning and impinging jet is also depicted. As shown in these figures, the model was found capable of reproducing wave breaking process and the validity of the model was confirmed in simulating the interaction of gas and liquid phases.

\begin{tabular}{|l|l|l|l|}
\hline \multicolumn{3}{|l|}{ Table 2. Numerical conditions (Test 2) } \\
\hline Computational domain & $540 \times 80[\mathrm{mesh}]$ & Water viscosity coefficient & $1.0 \times 10^{-3}[\mathrm{~Pa} \cdot \mathrm{s}]$ \\
\hline Mesh size & $\Delta x=0.30[\mathrm{~m}] \sim 0.01[\mathrm{~m}]$ & Air viscosity coefficient & $1.8 \times 10^{-5}[\mathrm{~Pa} \cdot \mathrm{s}]$ \\
\cline { 2 - 4 } & $\Delta z=0.02[\mathrm{~m}] \sim 0.005[\mathrm{~m}]$ & Surface tension coefficient & $7.2 \times 10^{-2}[\mathrm{~N} / \mathrm{m}]$ \\
\hline Time step interval & $0.0001[\mathrm{~s}]$ & Gravitational acceleration & $9.80665\left[\mathrm{~m} / \mathrm{s}^{2}\right]$ \\
\hline Water density & $1000.0\left[\mathrm{~kg} / \mathrm{m}^{3}\right]$ & Atmospheric pressure & $1013[\mathrm{hPa}]$ \\
\hline Air density & $1.20\left[\mathrm{~kg} / \mathrm{m}^{3}\right]$ & & \\
\hline
\end{tabular}
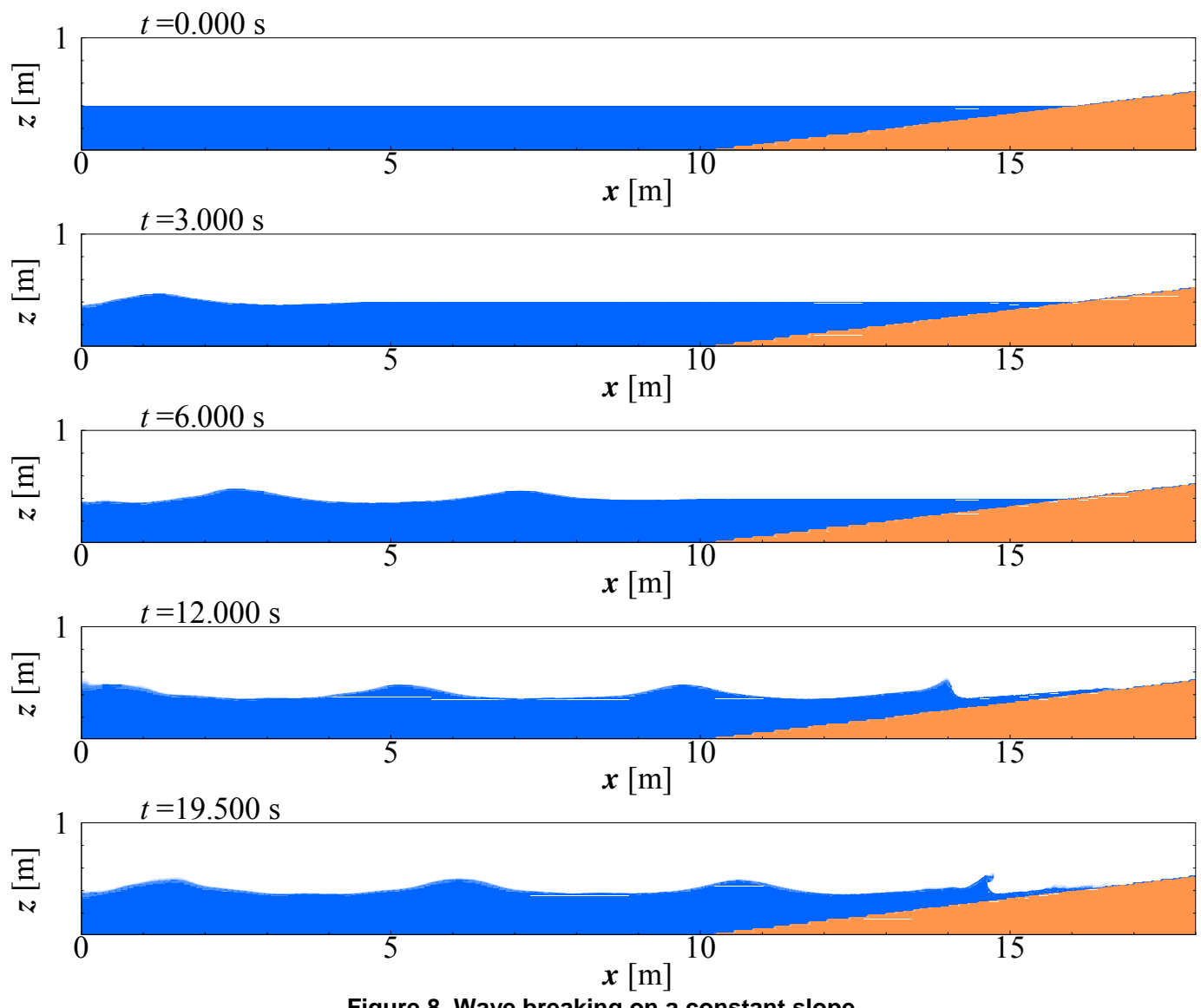

Figure 8. Wave breaking on a constant slope 

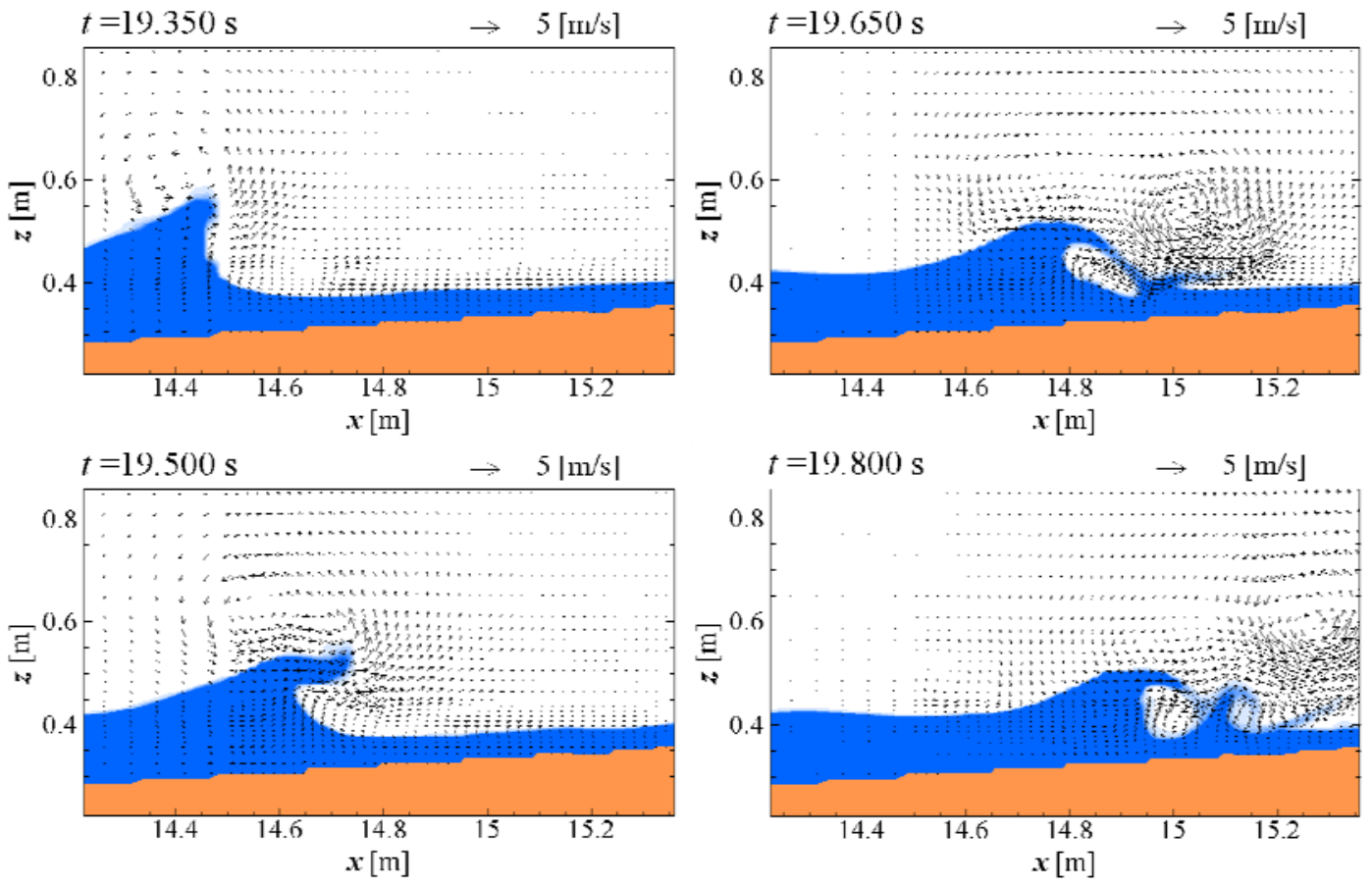

Figure 9. The velocity field in x-z plan around the breaking zone

\section{Dynamic Behavior of a Rigid Floating Body under Wave Action}

Dynamic analysis of a movable body under wave action would be considered as one of the most difficult problems in numerical simulations because advanced techniques are required to treat moving boundaries as well as nonlinear interaction among three phases of gas, liquid and solid. Here the proposed model is applied to the dynamic behavior of a rigid floating body under wave action on a slope to confirm its validity in solving the above-mentioned challenges.

Fig. 10 shows the initial condition for the simulation test. The wave generation source was located at $x=0.0 \mathrm{~m}$, and an energy dissipation zone was set up from $x=-6.0 \mathrm{~m}$ to $x=0.0 \mathrm{~m}$. A constant gradient of $1 / 15$ was set for a slope. Wave period and wave height are $1.5 \mathrm{~s}$ and $0.1 \mathrm{~m}$, respectively. A rigid body was set afloat over the slope as indicated in Fig. 10. The numerical conditions are shown in Table 3.

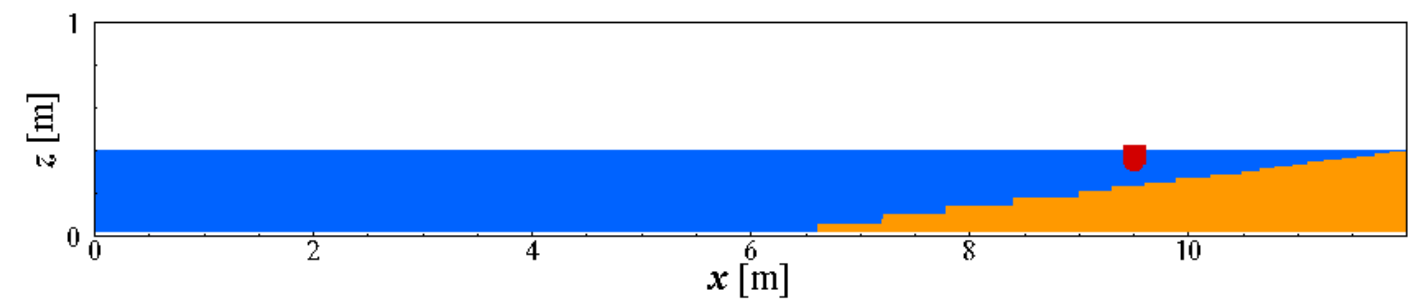

Figure 10. Initial condition for numerical simulation of dynamic behavior of a rigid floating body

\begin{tabular}{|l|l|l|l|}
\hline \multicolumn{4}{|l|}{ Table 3. Numerical conditions (Test 3) } \\
\hline Computational domain & $900 \times 50[\mathrm{mesh}]$ & Solid density & $800\left[\mathrm{~kg} / \mathrm{m}^{3}\right]$ \\
\hline Mesh size & $\Delta x=0.02[\mathrm{~m}] \sim 0.01[\mathrm{~m}]$ & Water viscosity coefficient & $1.0 \times 10^{-3}[\mathrm{~Pa} \cdot \mathrm{s}]$ \\
\cline { 2 - 4 } & $\Delta z=0.05[\mathrm{~m}] \sim 0.01[\mathrm{~m}]$ & Air viscosity coefficient & $1.8 \times 10^{-5}[\mathrm{~Pa} \cdot \mathrm{s}]$ \\
\hline Time step interval & $0.0001[\mathrm{~s}]$ & Surface tension coefficient & $7.2 \times 10^{-2}[\mathrm{~N} / \mathrm{m}]$ \\
\hline Water density & $1000.0\left[\mathrm{~kg} / \mathrm{m}^{3}\right]$ & Gravitational acceleration & $9.80665\left[\mathrm{~m} / \mathrm{s}^{2}\right]$ \\
\hline Air density & $1.20\left[\mathrm{~kg} / \mathrm{m}^{3}\right]$ & Atmospheric pressure & $1013[\mathrm{hPa}]$ \\
\hline
\end{tabular}



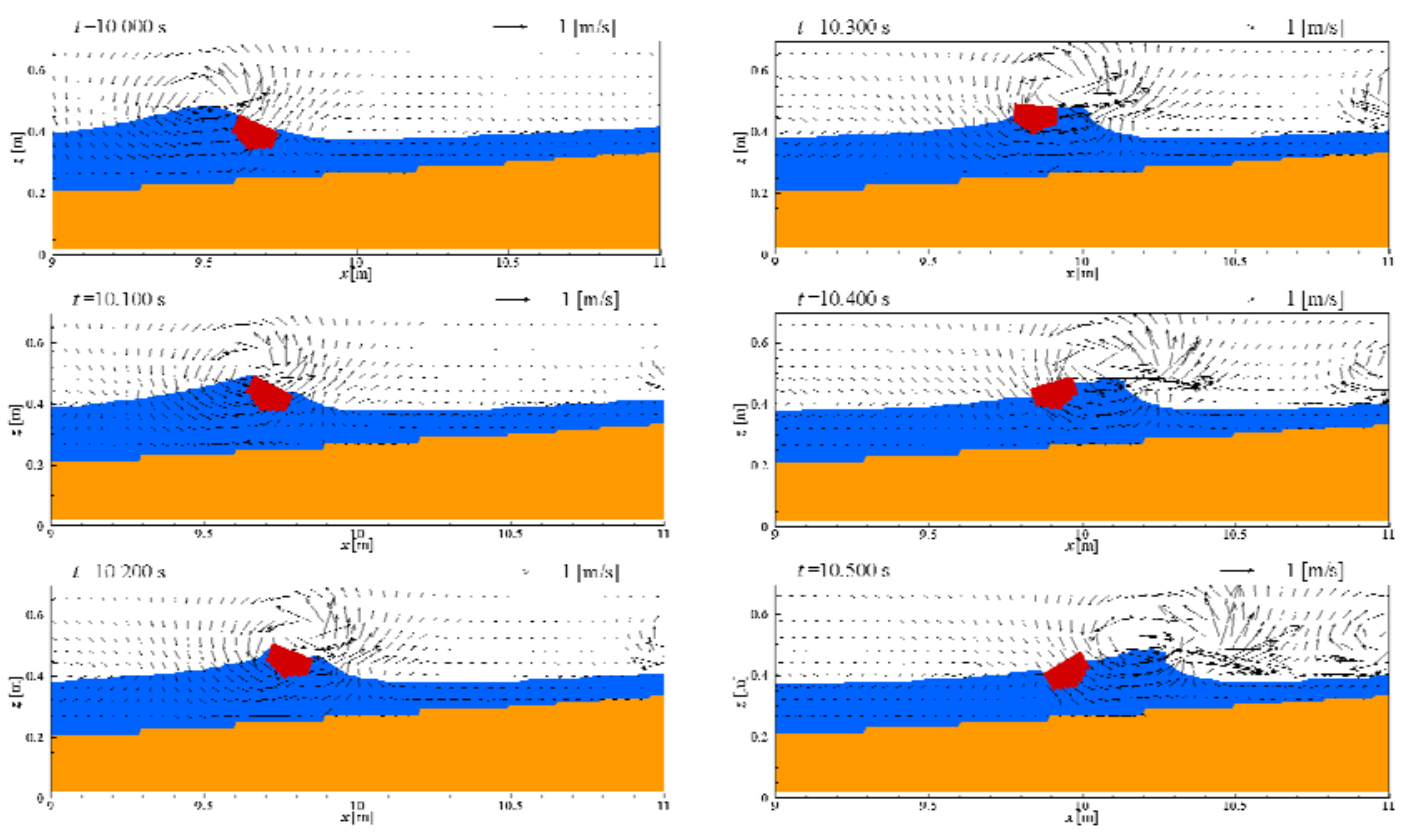

Figure 11. Dynamic behavior of a rigid floating body on the slope under wave action

Fig. 11 gives the numerical results of the dynamic behavior of the rigid floating body under wave action. After the generated wave hit the floating body, the body was turned and moved onshore by the wave forces. The configuration and volume of the body are also found to be preserved at any time. The numerical results, therefore, revealed the validity of the model in simulating the interaction of gas, liquid and solid phases.

\section{Collapse Simulation of Bingham Fluid Column}

Hereafter the model is applied to the collapse phenomenon of a Bingham fluid column and the interaction of a Bingham fluid column and two rigid bodies in order to confirm its validity.

First, two numerical simulations of the collapse phenomena of a Bingham and Newtonian fluid columns were conducted to verify the validity of the constitutive law of non-Newtonian fluid proposed in the model. Both the computational domains were taken as $0.75 \mathrm{~m}$ and $0.4 \mathrm{~m}$ in the directions of $x$ and $z$ respectively. A configuration of both the fluid columns was a rectangle of $0.1 \mathrm{~m}$ wide and $0.2 \mathrm{~m}$ high. The wall boundary condition for velocity was the slip condition. The velocity was initially set at zero in the entire computational domains. Other numerical conditions are shown in Table 4.

Fig. 12 shows the time variation of the gas-liquid interface and velocity field after the collapses of the columns. The counterclockwise circulation flows take place in both the computational domains by the collapses of the fluid columns due to gravity force. The magnitude of the velocities around the gasliquid interface in the simulation of the collapse of Bingham fluid column, however, is much less as compared to the case of Newtonian fluid column. Consequently, after $0.4 \mathrm{~s}$ from the start of computation, the Newtonian liquid collides with the right wall whereas the Bingham liquid only reaches the location around $x=0.2 \mathrm{~m}$. Numerical results, therefore, demonstrated a strong influence of the constitutive law of fluid on the fluid dynamics and confirmed the validity of the model for simulating the non-Newtonian fluid as well as the Newtonian fluid.

\begin{tabular}{|l|l|l|l|}
\hline Table 4. Numerical conditions (Test 4) \\
\hline Computational domain & $150 \times 80[\mathrm{mesh}]$ & Water viscosity coefficient & $1.0 \times 10^{-3}[\mathrm{~Pa} \cdot \mathrm{s}]$ \\
\hline Mesh size & $\Delta x=0.005[\mathrm{~m}]$ & Air viscosity coefficient & $1.8 \times 10^{-5}[\mathrm{~Pa} \cdot \mathrm{s}]$ \\
\cline { 2 - 4 } & $\Delta z=0.005[\mathrm{~m}]$ & Surface tension coefficient & $7.2 \times 10^{-2}[\mathrm{~N} / \mathrm{m}]$ \\
\hline Time step interval & $0.0001[\mathrm{~s}]$ & Gravitational acceleration & $9.80665\left[\mathrm{~m} / \mathrm{s}^{2}\right]$ \\
\hline Bingham fluid density & $1000.0\left[\mathrm{~kg} / \mathrm{m}^{3}\right]$ & Atmospheric pressure & $1013[\mathrm{hPa}]$ \\
\hline Air density & $1.20\left[\mathrm{~kg} / \mathrm{m}^{3}\right]$ & Cohesion $c$ & $100.0[\mathrm{~Pa} \cdot \mathrm{s}]$ \\
\hline Solid density & $1000.0\left[\mathrm{~kg} / \mathrm{m}^{3}\right]$ & Angle of internal friction $\phi$ & $0.0[\mathrm{degree}]$ \\
\hline & & $\beta$ & 0.02 \\
\hline
\end{tabular}



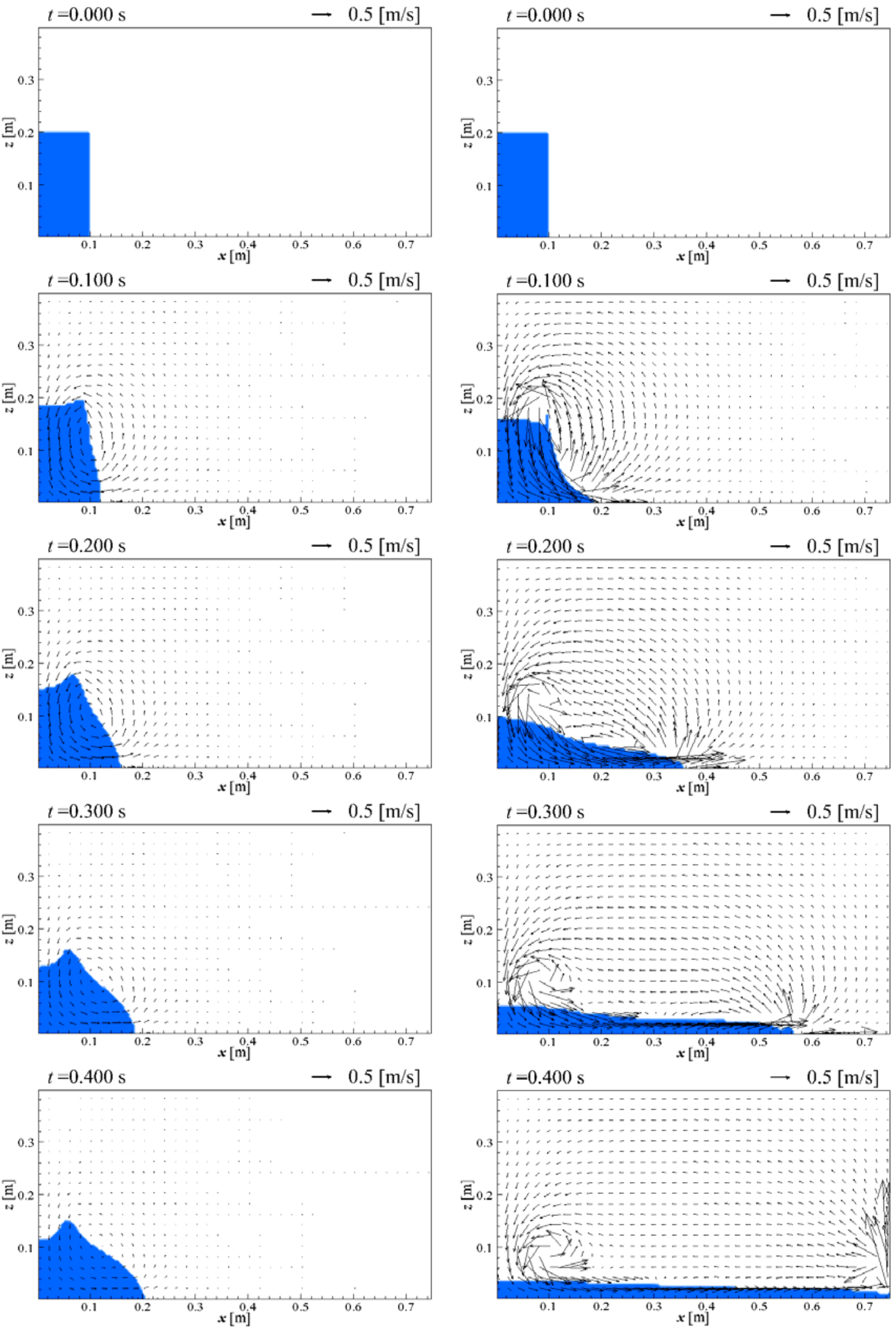

(a)Non-Newtonian fluid (Bingham fluid)

(b) Newtonian fluid

Figure 12. Dynamic behavior of non-Newtonian fluid and Newtonian fluid 

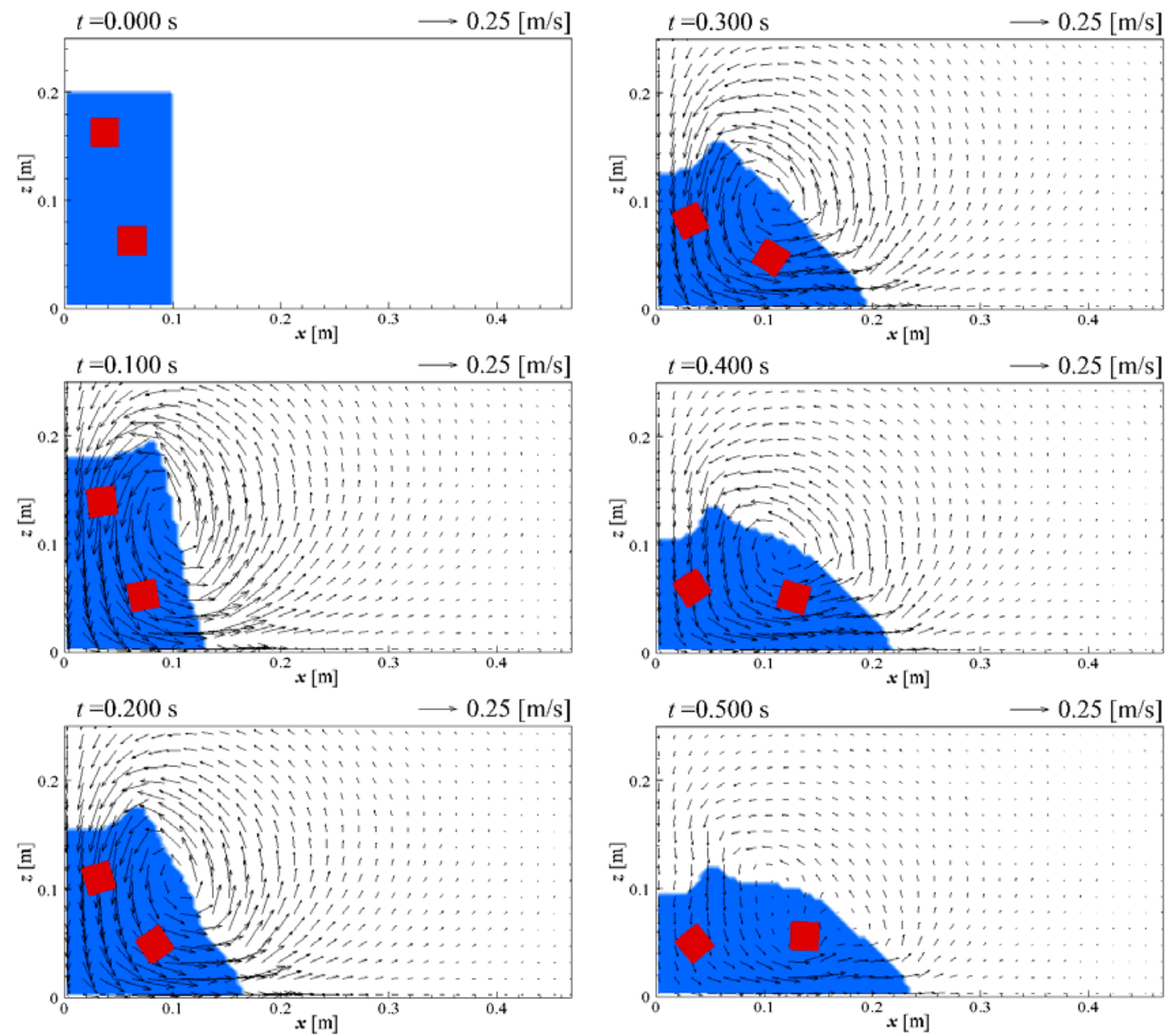

Figure 13. Interaction of Bingham fluid with two rigid bodies

Here the model is employed to simulate the interaction of Bingham fluid with two rigid bodies. In the computational domain, one rigid body and the other were set near the top and bottom of the column, respectively. Fig. 13 shows the time variation of the dynamic behavior of the two rigid bodies and the velocity field simultaneously. After the column collapsed, the counterclockwise circulation flow is found to take place. As the results, both the two rigid bodies were rotated counterclockwise. The two rigid bodies located at the top and bottom of the fluid column are confirmed to move downward and rightward respectively with the flow caused by the collapse of the fluid column. Thus, the validity of the model was verified for simulating the interaction of the non-Newtonian fluid and multiple rigid bodies.

\section{CONCLUSION}

A 2-D numerical wave flume with solid-gas-liquid interaction as well as the constitutive laws of Newtonian and non-Newtonian fluids was newly developed in this study. The validity and utility of the model was qualitatively and quantitatively verified throughout some applications to wave breaking and post-breaking wave deformation on the slope, the dynamic motion of a floating body under wave action and the collapse of the Bingham fluid column with multiple rigid bodies.

\section{ACKNOWLEDGMENTS}

This research is supported in part by Grant-in-Aid for Young Scientist (A) (Project No.21686046, Head Investigator: K. Kawasaki) of the Ministry of Education, Culture, Sports, Science and Technology, Japan. 


\section{REFERENCES}

Brackbill, J.U., D.B. Kothe, and C. Zemach. 1992. A continuum method for modeling surface tension, Journal of Computational Physics, 100, 335-354.

Brorsen, M., and J. Larsen. 1987. Source generation of nonlinear gravity waves with the boundary integral equation method, Coastal Engineering, 11, 93-113.

Cruz, E., H. Yokoki, M. Isobe, and A. Watanabe. 1993. An absorbing boundary condition for nonlinear waves, Proceedings of Coastal Engineering, JSCE, 40, 46-50 (in Japanese).

Kawasaki, K. 2005a. Numerical Model of 2-D Multiphase Flow with Solid-Liquid-Gas Interaction, International Journal of Offshore and Polar Engineering, 15(3), 198-203.

Kawasaki, K. 2005b. Numerical simulation of solid-gas-liquid phase flow in a three-dimensional field, Proceedings of 3rd International Conference on Asian and Pacific Coasts, 1868-1879.

Kawasaki, K., and M. Hakamata. 2006. Numerical analysis of time-changing wave force acting on drifting rigid structure with solid-gas-liquid phase flow model, Proceedings of the 30th International Conference on Coastal Engineering, 4507-4519.

Kawasaki, K., and N. Mizutani. 2007. Numerical simulation of bore-induced dynamic behavior of rigid body using 2-D multiphase flow numerical model, Proceedings of International Conference on Coastal Structures 2007, 1477-1488.

Kawasaki, K., and K. Ogiso. 2009. Development of 3-D multiphase flow numerical model "DOLPHIN-3D" and its application to wave-rigid body interaction problems, Proceedings of the 31 st International Conference on Coastal Engineering 2008, 3199-3211.

Moriguchi S., A.Yashima, K. Sawada, R. Uzuoka, and M. Ito. 2005. Numerical simulation of flow failure of geomaterials based on fluid dynamics, Solids and foundations, 45(2), 155-165.

Nakamura, T., R. Tanaka, T. Yabe, and K. Takizawa. 2001. Exactly conservative semilagrangian scheme for multi-dimensional hyperbolic equations with directional splitting technique, Journal of Computational Physics, 174, 171-207.

Salvetti, M.V., and S. Banerjee. 1995. A priori tests of a new dynamic subgrid-scale model for finitedifference large-eddy simulations, Physics of Fluids, 7(11), 2831-2847.

Xiao, F., T. Yabe, T. Ito, and M. Tajima. 1997. An algorithm for simulating solid objects suspended in stratified flow, Computer Physics Communications, 102, 147-160.

Yabe, T., and T. Aoki. 1991. A universal solver for hyperbolic equations by cubic-polynominal interpolation I. One-dimensional solver, Computer Physics Communications, 66, 219-232.

Yabe, T., and R. Tanaka, T. Nakamura, and F. Xiao. 2001. An Exactly Conservative Semi-Lagrangian Scheme (CIP-CSL) in One Dimension, Monthly Weather Review, 129, 332-344.

Yamada, Y., T. Oshiro, and Y. Masuda. 1998. Application of MAC method to flow analysis of fresh concrete, Annual Journal of Concrete Engineering, 20(1), 131-136 (in Japanese). 